\author{
Jonas Nielsen \\ Manja Nilsson \\ Filip Fredén \\ Jan Hultman \\ Ulrica Alström \\ Jesper Kjærgaard \\ Göran Hedenstierna \\ Anders Larsson
}

\section{Central hemodynamics during lung recruitment maneuvers at hypovolemia, normovolemia and hypervolemia. A study by echocardiography and continuous pulmonary artery flow measurements in lung-injured pigs}

Published online: 5 May 2006

(C) Springer-Verlag 2006

The online version of the original article can be found at http://dx.doi.org/10.1007/s00134-006-0082-0

J. Nielsen (

Copenhagen University Hospital, Gentofte, Department of

Anesthesia and Intensive Care Medicine,

Niels Andersensvej 65, 2900 Hellerup, Denmark

e-mail: Jonie@gentoftehosp.kbhamt.dk

Tel.: +45-39-773941

Fax: +45-39-777658

M. Nilsson · F. Fredén · J. Hultman · U. Alström

Uppsala University Hospital, Department of Anesthesia and

Intensive Care Medicine,

Uppsala, Sweden

J. Kjærgaard

Copenhagen University Hospital, Rigshospitalet, Department of

Cardiology,

Copenhagen, Denmark

J. Nielsen $\cdot$ G. Hedenstierna

Uppsala University Hospital, Department of Clinical Physiology,

Uppsala, Sweden

\section{A. Larsson}

Aalborg Hospital, Aarhus University Hospital, Department of

Anesthesia and Intensive Care Medicine and Center for

Cardiovascular Research,

Aalborg, Denmark

\section{Intensive Care Med (2006) 32:585-594}

Dr. Jonas Nielsen and co-authors would like to make a correction in Table 1.

They have by mistake used the sort "\%" for eccentricity index within the table. The values are in absolute numbers and "\%" is deleted. 


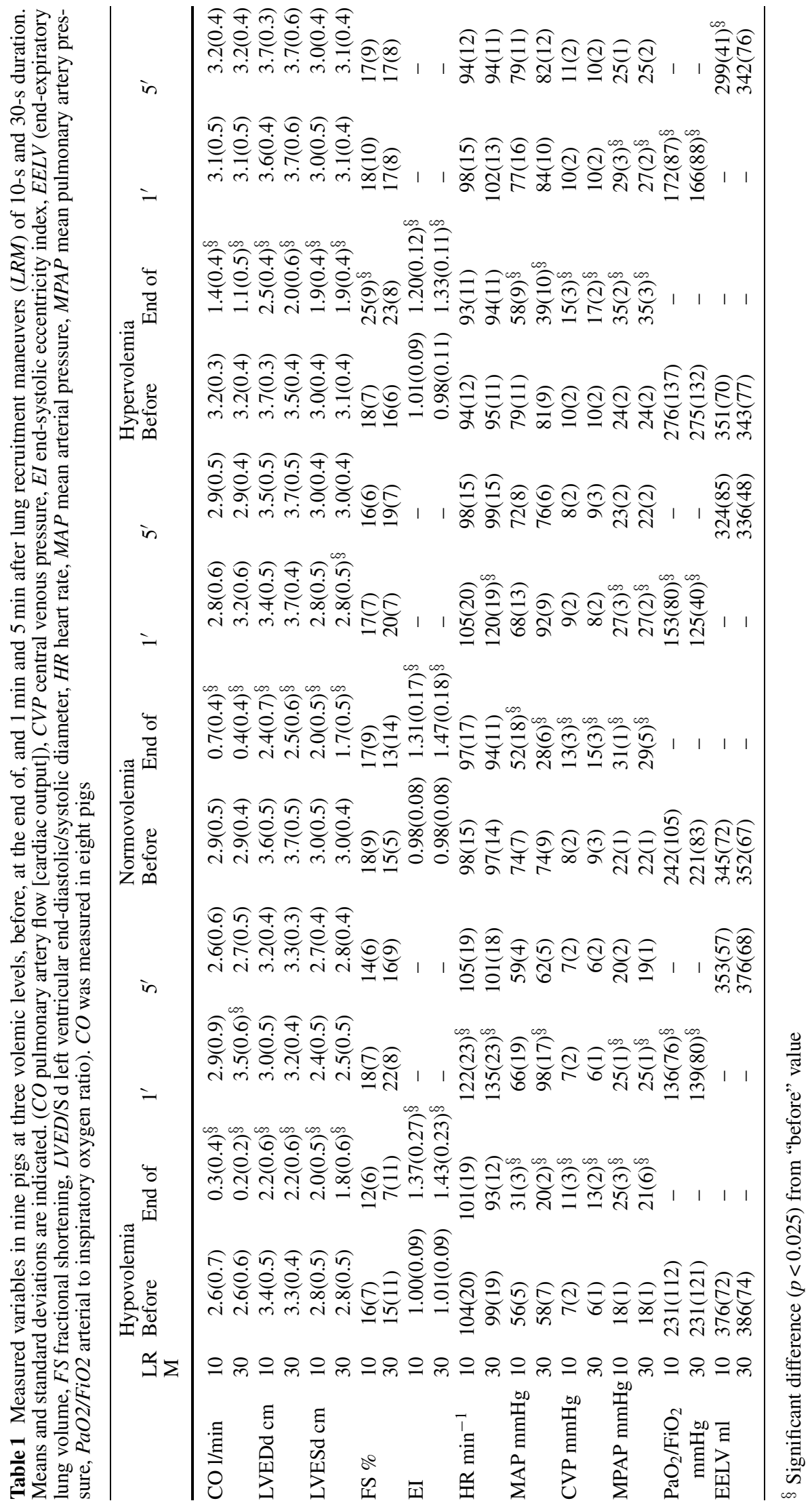

